\title{
Updated collisional probabilities of minor body populations
}

\author{
A. Dell'Oro ${ }^{1}$, F. Marzari ${ }^{2}$, P. Paolicchi ${ }^{3}$, and V. Vanzani ${ }^{4}$ \\ 1 Dipartimento di Fisica, Università di Pisa, piazza Torricelli 2, 56127 Pisa, Italy \\ 2 Dipartimento di Fisica, Università di Padova, Via Marzolo 8, 35131 Padova, Italy \\ e-mail: francesco.marzari@pd.infn.it \\ 3 Dipartimento di Fisica, Università di Pisa, piazza Torricelli 2, 56127 Pisa, Italy \\ e-mail: paolo@astr15pi.difi.unipi.it \\ 4 Dipartimento di Fisica, Università di Padova, Via Marzolo 8, 35131 Padova, Italy \\ e-mail: vanzani@pd.infn.it
}

Received 5 June 2000 / Accepted 31 August 2000

\begin{abstract}
The consistent increase in the discovery rate of new asteroids and Trans Neptunian Objects (TNOs) in these last years has urged an update of the values of intrinsic probability of collision and impact velocity for some minor body populations. With the statistical method of Dell'Oro \& Paolicchi (1998), we have recomputed the values of impact probability and velocity for Hilda asteroids, for Trojans vs. Short Period Comets (SPC), and for TNOs. The algorithm of Dell'Oro and Paolicchi is particularly suited for the task since it can account for resonant behaviour (Dell'Oro et al. 1998) and for the clustering of the perihelion longitude of Main Belt asteroids and Hildas, caused by the presence of a forced component in the eccentricity. The Hilda population turns out to be well sampled in the orbital parameter space since no significant changes are found for the collision frequency among Hildas, and of Hildas with Main Belt asteroids, although a much larger sample of orbits has been used in our computations (232 objects) vs. the smaller group used in previous computation by Dahlgren (1998) (40 objects). We also computed the impact rate of SPCs vs. Trojans that turned out to be an order of magnitude lower respect to the Trojans vs. Trojans impact rate. The relative velocity is instead about $30 \%$ higher. Using reasonable estimates of SPC and Trojan number densities, we find that approximately 1 every 100 collisions involving Trojans may be with an SPC. In the case of TNOs there is a consistent discrepancy between our values of the collision probability and impact speed, and those computed by Davis \& Farinella (1997). The consistent increase in the number of known TNOs (186 at present, only 16 at the time of the Davis and Farinella's work) has led to a better knowledge of their distribution in the phase space and, consequently, to more reliable estimates of the collisional probability and impact velocity.
\end{abstract}

Key words. minor planets - comets - Kuiper Belt

\section{Introduction}

The present size distributions of Main Belt asteroids, Trojans and TNOs have been shaped from the primordial ones by collisional processes ongoing over the whole age of the Solar System. Studies of the collisional evolution of these populations require two essential pieces of information: the frequency of collisional events and the characteristic impact speed. Both these quantities depend on the distribution of the minor body population in the phase space of the orbital elements. Quantitative estimates of the intrinsic probability of collision, that is the average impact rate per unit time and per unit cross-sectional area of the colliding bodies, and of the mean impact velocity have been derived either with methods based on the

Send offprint requests to: A. Dell'Oro, e-mail: aldo@astr15pi.difi.unipi.it direct numerical integration of the orbits (Marzari et al. 1996, 1997; Dahlgren 1998) or with statistical algorithms (Wetherill 1967; Greenberg 1982; Farinella \& Davis 1992; Vedder 1996; Dell'Oro \& Paolicchi 1998). The basic assumption that validates both these methods is that the set of orbits used in the computation is representative of the distribution of the whole population in the orbit parameter space.

For populations of minor bodies that are undersampled or affected by observational bias, the estimates of the intrinsic probability $\left\langle P_{i}\right\rangle$ and mean impact velocity $\left\langle V_{i}\right\rangle$ must be updated as additional observations and discoveries enrich the known population. This process should lead, for increasingly larger and more complete sets, to converging values. In this optics, the statistical approach should be preferred since it allows to update the values of $\left\langle P_{i}\right\rangle$ and $\left\langle V_{i}\right\rangle$ with a very short computational effort 
compared to the long and cumbersome numerical integrations required by the numerical models. This updating process is particularly relevant for Trojans and TNOs that, at present, are still poorly known and incomplete.

In this paper we concentrate on these populations, for which we compute updated values of $\left\langle P_{i}\right\rangle$ and $\left\langle V_{i}\right\rangle$. The number of Hilda asteroids known at present has grown by a factor 6 relative to the number of objects used by Dahlgren (1998) in his numerical integrations. However, we find that the values of $\left\langle P_{i}\right\rangle$ and of $\left\langle V_{i}\right\rangle$ computed over a large sample of 232 objects are not significantly different from the previous estimates. It means that the small sample of 40 asteroids used by Dahlgren was already covering a significant fraction of the volume in the phase space occupied by the entire Hilda population. Since 1997, an increasing number of TNOs' have been discovered (almost 200) so that the set of representative orbits for computing $\left\langle P_{i}\right\rangle$ and $\left\langle V_{i}\right\rangle$ has grown by more than an order of magnitude compared to the number of objects (16) known at the time of the work of Davis \& Farinella (1997). The hypothetical population we derive for the inner Kuiper Belt, after accounting for the strong observational bias, is more complete and allows a better determination of both $\left\langle P_{i}\right\rangle$ and $\left\langle V_{i}\right\rangle$. Marked differences appear between our estimates of both the collision probability and impact velocity compared to those of Davis and Farinella's. The probability $\left\langle P_{i}\right\rangle$ is a factor 10 lower while the average impact speed $\left\langle V_{i}\right\rangle$ is more than twice larger. This suggests the possibility of an update of the collisional evolution simulations for the Kuiper Belt. We compute also the probability of collisions of SPCs with Trojan asteroids, that was never estimated before. The impact speed is high compared to that of intra-Trojans encounters, but the collisional probability is an order of magnitude lower.

The paper is structured as follows: the numerical algorithm is summarized in Sect. 2. In the same section we detail how in the statistical model we deal with resonances and the clustering in the perihelion longitudes caused by the forced eccentricity. Section 3 is devoted to the presentation of the updated values of $\left\langle P_{i}\right\rangle$ and $\left\langle V_{i}\right\rangle$ for Hildas, Trojans, and SPCs. In Sect. 4 we present the new results for TNOs.

\section{Numerical algorithm}

To compute the intrinsic collision probability and velocities for different minor body populations, we have applied the statistical algorithm developed by Dell'Oro \& Paolicchi (1998). This algorithm allows to overcome the implicit assumptions of Wetherill's theory of a uniform density for the orbital angles and it allows to handle complex dynamical situations like resonance trapping (Hildas, Trojans, and Plutinos) and the non-uniform distribution of the perihelion longitudes of Main Belt asteroids caused by secular perturbations. In the next two sections we will describe how the original formulation of the statistical model of Dell'Oro and Paolicchi has been modified to take into account these two dynamical phenomena.

\subsection{How to handle resonances}

The common feature of Hildas, Trojans, and of some of the TNOs is their resonant behaviour. Hilda asteroids are locked in a 3:2 mean motion resonance with Jupiter, Trojans are in a 1:1 resonance with Jupiter, and a significant fraction of TNOs, the Plutinos, are in a 3:2 mean motion resonance with Neptune. For this reason $\left\langle P_{i}\right\rangle$ cannot be computed with simple methods based on the Wetherill's formalism (Wetherill 1967) since the angular orbital variables are not randomly distributed, but are mutually related through the critical angle. As a consequence, the phase space available for the motion is reduced and the impact probability increased. The algorithm of Dell'Oro \& Paolicchi (1998) is particularly suited to compute the collision probability among bodies in resonance.

We briefly summarize here the main aspects of the algorithm and why it is particularly suited for resonant orbits. The basic formula for the rate of collision between two given objects is:

$n_{\mathrm{T}}=\pi \tau^{2} \int_{f_{0}, \omega_{0}, \Omega_{0}} \Delta\left(f_{0}, \omega_{0}, \Omega_{0}, f_{\mathrm{c}}, \omega_{\mathrm{c}}, \Omega_{\mathrm{c}}\right) \frac{U}{|J|} \mathrm{d} f_{0} \mathrm{~d} \omega_{0} \mathrm{~d} \Omega_{0} .(1)$

The orbital elements of the target $a_{0}, e_{0}$ and $\sin I_{0}$, and the corresponding $a, e$, and $\sin I$ of the projectile, do not appear explicitly in the formula, but are required in the computation and are assumed to be constant. The integration is performed on the angular elements of the target $f_{0}, \omega_{0}, \Omega_{0}$. The quantities $f_{\mathrm{c}}, \omega_{\mathrm{c}}, \Omega_{\mathrm{c}}$ are the angular elements of the projectiles at the instant of the impact. The relative impact velocity $U$ is computed from these quantities.

The basic feature of the algorithm for resonant orbits is the function $\Delta$ that describes the statistical distribution in the 6 -D space of the coordinates $f_{0}, \omega_{0}, \Omega_{0}, f, \omega, \Omega$ of the target-projectile couple. For non-resonant cases, this function can be factorized by separating the variables of the target from the variables of the projectile since they are not correlated. When resonant orbits are considered, the motion of the bodies are correlated to that of Jupiter (or Neptune for Plutinos) and the target evolution cannot be separated from that of the projectile.

We devised the following approach to build up the function $\Delta$. We imagined the 6-D space of the orbital elements embedded in a $(6+N)$-D space that includes $N$ angular variables of Jupiter (or Neptune). For mean motion resonances, $N=1$ and the variable to add is the longitude of Jupiter $\lambda_{\mathrm{J}}$. In $\Delta$ we have to account for the instantaneous keplerian motion of the bodies around the Sun, so $\Delta$ includes the following normalized form (Dell'Oro \& Paolicchi 1998):

$$
\begin{aligned}
\Delta_{k}= & \delta_{k}(f) \delta_{k}\left(f_{0}\right)=\frac{1}{(2 \pi)^{3}} \frac{\left(1-e^{2}\right)^{3 / 2}}{(1+e \cos f)^{2}} \\
& \times \frac{1}{(2 \pi)^{3}} \frac{\left(1-e_{0}^{2}\right)^{3 / 2}}{\left(1+e_{0} \cos f_{0}\right)^{2}} .
\end{aligned}
$$

We then assume that the critical argument of the resonance can be described as a harmonic oscillator. 
The probability of finding the angle between two values $x$ and $x+\mathrm{d} x$ is:

$P_{A}(x)=\left\{\begin{array}{cr}0 & |x|>A \\ (1 / \pi A)\left(1-x^{2} / A^{2}\right)^{-1 / 2} \mathrm{~d} x & |x|<A\end{array}\right.$

where $A$ is the amplitude of the oscillator. To model in the function $\Delta$ the relation among the angular variables of target and projectile induced by the resonance, we weight the function with a coefficient proportional to $\Phi_{A}(\sigma)=$ $2 \pi P_{A}(\sigma)$ where $\sigma$ is the critical angle of the resonance, $A$ the libration amplitude, and $2 \pi$ a normalization factor (Dell'Oro \& Paolicchi 1998). We have adopted the same formalism already used by Dell'Oro et al. (1998) for the study of the collisions among Trojans. For Hildas, Trojans, and Plutinos, both target and projectile are in resonance, so the function $\Delta$ has the following form:

$\Delta=\Delta_{k} \Phi_{A}\left(\sigma_{0}\right) \Phi_{A}(\sigma)$

where

$\sigma_{0}=3 \lambda_{\mathrm{J}}-2 \lambda_{0}-\tilde{\omega}_{0} \quad \sigma=3 \lambda_{\mathrm{J}}-2 \lambda-\tilde{\omega}$

for Hildas,

$\sigma_{0}=\lambda_{\mathrm{J}}-\lambda_{0} \quad \sigma=\lambda_{\mathrm{J}}-\lambda$

for Trojans, and

$\sigma_{0}=2 \lambda_{\mathrm{N}}-3 \lambda_{0}-\tilde{\omega}_{0} \quad \sigma=2 \lambda_{\mathrm{N}}-3 \lambda-\tilde{\omega}$

for Plutinos. $\lambda_{0}$ and $\lambda$ refer to the mean longitudes of the target and projectile, respectively. We remind that $\Delta$ depends also on the external parameter $\lambda_{\mathrm{J}}$, so in computing the probability the integration must also run over $\lambda_{\mathrm{J}}$. The final equation is:

$$
\begin{aligned}
& n_{\mathrm{T}}=\pi \tau^{2} \frac{1}{2 \pi} \\
& \quad \times \int_{f_{0}, \omega_{0}, \Omega_{0}, \lambda_{\mathrm{J}}} \Delta\left(f_{0}, \omega_{0}, \Omega_{0}, f_{\mathrm{c}}, \omega_{\mathrm{c}}, \Omega_{\mathrm{c}}\right) \frac{U}{|J|} \mathrm{d} f_{0} \mathrm{~d} \omega_{0} \mathrm{~d} \Omega_{0} \mathrm{~d} \lambda_{\mathrm{J}}
\end{aligned}
$$

where the factor $1 / 2 \pi$ is required in order to obtain the mean value with respect to $\lambda_{\mathrm{J}}$.

Since we consider populations of minor bodies that not only collide with members of their same group but can interact with other groups, we summarize here the forms used for the function $\Delta$ in the different cases considered:

$$
\begin{array}{lc}
\text { HH, PP } & \Delta=\Delta_{k} \Phi_{\Delta \sigma_{0}}\left(\sigma_{0}\right) \Phi_{\Delta \sigma}(\sigma) \\
\mathrm{TT} & \Delta=\Delta_{k} \Phi_{\Delta \lambda_{0}}\left(\lambda_{0}-\lambda_{\mathrm{L}}\right) \Phi_{\Delta \lambda}\left(\lambda-\lambda_{\mathrm{L}}\right) \\
\mathrm{HT} & \Delta=\Delta_{k} \Phi_{\Delta \sigma_{0}}\left(\sigma_{0}\right) \Phi_{\Delta \lambda}\left(\lambda-\lambda_{\mathrm{L}}\right)
\end{array}
$$

$\mathrm{TM}, \mathrm{TC} \quad \Delta=\Delta_{k} \Phi_{\Delta \lambda_{0}}\left(\lambda_{0}-\lambda_{\mathrm{L}}\right)$

$\mathrm{PK} \quad \Delta=\Delta_{k} \Phi_{\Delta \sigma_{0}}\left(\sigma_{0}\right)$

where $\mathrm{H}$ stays for Hildas, $\mathrm{T}$ for Trojans, $\mathrm{M}$ for Main Belt asteroids, $\mathrm{C}$ for comets, $\mathrm{P}$ for Plutinos, $\mathrm{K}$ for TNOs' not in the 3:2 resonance. $\lambda_{\mathrm{L}}$ is the longitude of the Lagrangian point for the particular Trojan swarm under consideration.
Main Belt $(D>30$ km)

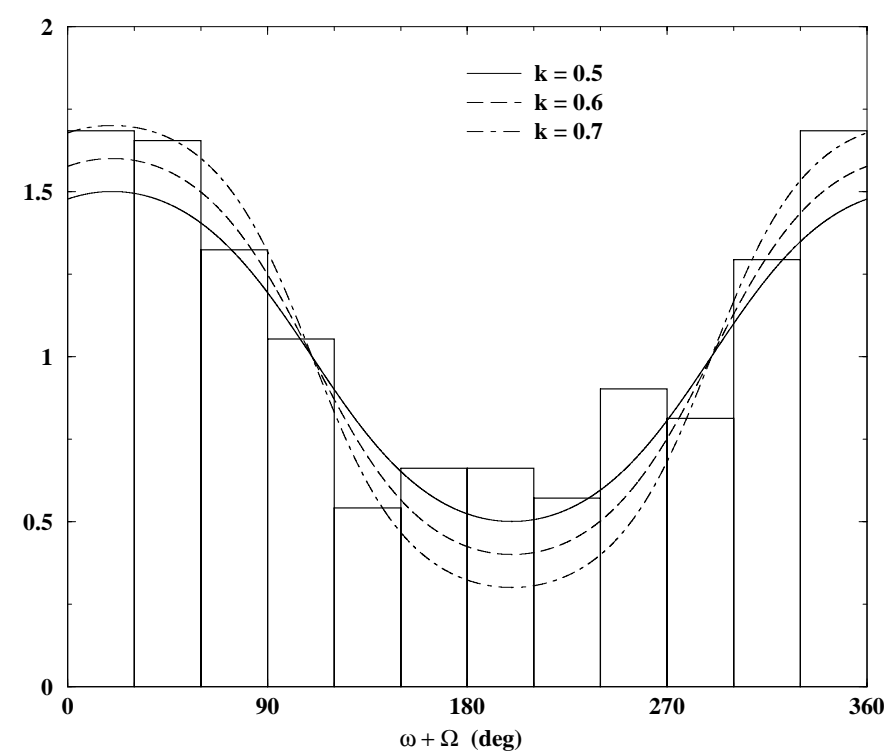

Fig. 1. Distribution of the longitudes of the perihelion for the Main Belt asteroids. The epoch is $21 / 2 / 97$

\subsection{The clustering in $\tilde{\omega}$ caused by secular perturbations}

The secular perturbations of the planets, principally of Jupiter, introduces an additional phenomenon that was not previously taken into account in the statistical methods based on Wetherill's formalism. According to Lagrange's theory, the secular perturbations causes the apsidal and nodal longitudes to precess over intervals of time of the order of $10^{4} \sim 10^{5}$ years. For models à la Wetherill the assumption of a uniform distribution, and then of a regular precession, is needed to compute averages over time of the impact configurations. However, the precession is only grossly regular since the forced component in the eccentricity of the asteroids due to Jupiter causes a reduction of the precession speed of the asteroid $\tilde{\omega}$ in the proximity of Jupiter's $\tilde{\omega}$. This effect generates a non-uniform distribution of the perihelion longitudes of the Main Belt asteroids, noted for the first time by Kresák (1967).

In Fig. 1 we show the observed distribution of the $\tilde{\omega}$ s of all Main Belt asteroids larger than $30 \mathrm{~km}$ (to avoid selection effects) in the form of a histogram. All the orbits are referred to a common epoch. The distribution is peaked at about $18^{\circ}$, the value of Jupiter's $\tilde{\omega}$ at the same epoch. For the Hildas a similar distribution is obtained (Fig. 3) although resonant perturbations can alter the precession speed of the perihelia. This clustering of the $\tilde{\omega}$ s was not taken into account in the previous statistical computations (Farinella \& Davis 1992; Bottke et al. 1994; Vedder 1996) and it may have a significant effect on the impact probability by favouring some geometrical configurations among the orbits of the bodies. We model the clustering by introducing in the statistical algorithm a suitable 


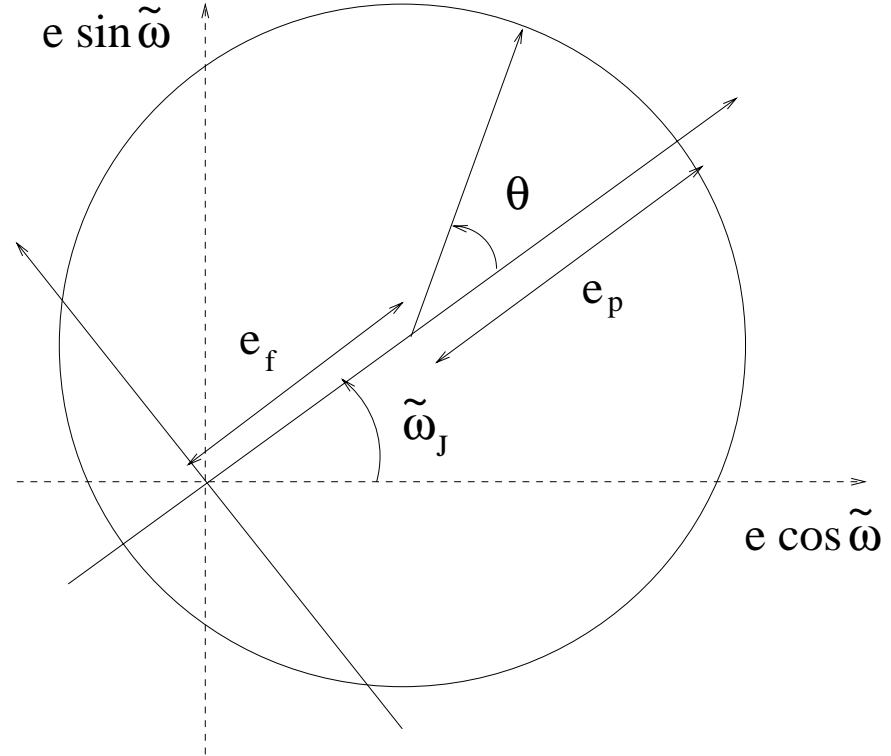

Fig. 2. First order Lagrange's theory of motion of the asteroids subjected to the gravitational influence of the Sun and Jupiter (see text)

\section{Hilda}

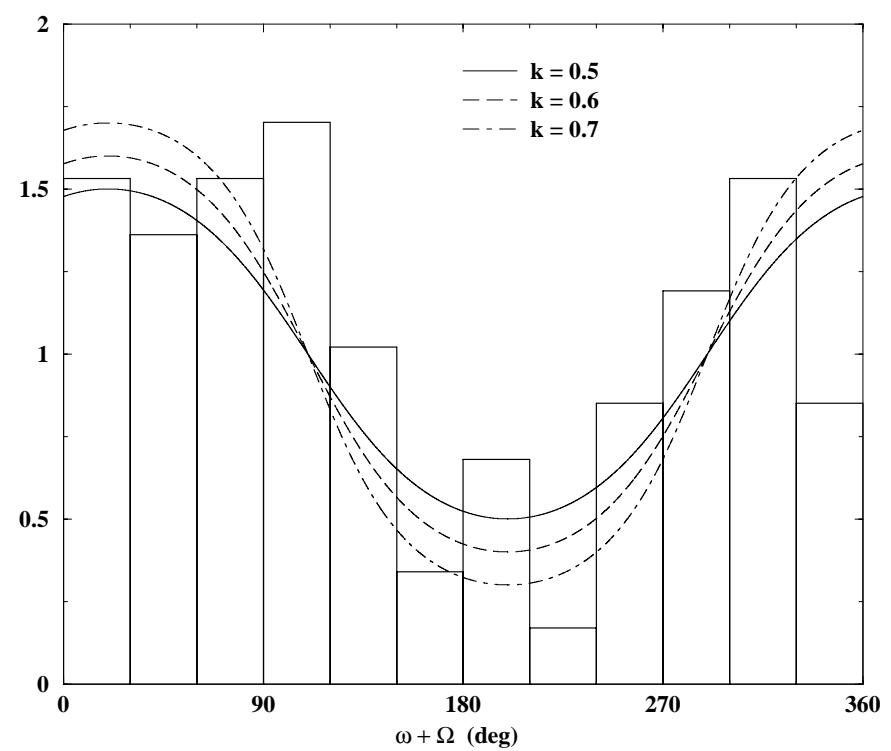

Fig. 3. Distribution of the longitudes of the perihelion for the Hildas. The epoch is $21 / 2 / 97$

function $\mathcal{L}$ that reproduces the observed distribution of $\tilde{\omega}$ for a fixed value of $\tilde{\omega}_{\text {J }}$. Our starting point in the construction of $\mathcal{L}$ is the first order Lagrange's theory.

In Fig. 2 we illustrate graphically in the plane $e \cos \tilde{\omega}$ $e \sin \tilde{\omega}$ the geometrical aspects of the Lagrange's theory. In the plane $e \cos \tilde{\omega}-e \sin \tilde{\omega}$ the motion of the asteroid is approximately described by a circle. The secular perturbations of Jupiter (we neglect at a first approximation the contribution of the other planets) induces a shift of the center of the circle from the origin of the coordinates to the top of a vector whose magnitude is the forced eccen- tricity $e_{\mathrm{f}}$, while the versus is given by Jupiter's $\tilde{\omega}$. This behaviour is well described in Greenberg (1975) and (1977). The Lagrange's theory requires that the angle $\theta$ circulates uniformly in time. The orbital angle $\alpha=\tilde{\omega}-\tilde{\omega}_{\mathrm{J}}$ is a nonlinear function of $\theta$ so it precesses with non-uniform speed depending on the value of $\theta$. From geometrical considerations, we can derive the following equation for the circulation velocity of $\alpha$ as a function of the constant precession rate of $\theta$ :

$\frac{\mathrm{d} \theta}{\mathrm{d} t}=\left[1+\frac{k \cos \alpha}{\sqrt{1-k^{2} \sin ^{2} \alpha}}\right] \frac{\mathrm{d} \alpha}{\mathrm{d} t}$

where $k$ is equal to the ratio between the forced and proper eccentricity. We consider here only the case of circulation when $e_{\mathrm{f}} \leq e_{\mathrm{p}}$, i.e. $k \leq 1$. This case covers the majority of asteroidal orbits in the Main Belt. The function that describes the distribution of the angle $\alpha$, assuming a uniform distribution for $\theta$ between $0-2 \pi$, is then:

$\mathcal{L}(\alpha)=\frac{1}{2 \pi}\left[1+\frac{k \cos \alpha}{\sqrt{1-k^{2} \sin ^{2} \alpha}}\right]$

where $2 \pi$ is introduced to normalize the density function $\mathcal{L}(\alpha)$.

The orbits of the asteroids in the Main Belt have different values of the ratio $e_{\mathrm{f}} / e_{\mathrm{p}}$, so we should combine many functions $\mathcal{L}(\alpha)$ with different values of $k$. In alternative, we can try to match the observed distribution of $\alpha=\tilde{\omega}-\tilde{\omega}_{\mathrm{J}}$ with a single function $\mathcal{L}(\alpha)$ and derive the value of the parameter $k$ from the fit. In Fig. 1 we show different fits to the observed distributions of $\tilde{\omega}$ with $\mathcal{L}$ using different values of $k$. The best fit for the Main Belt asteroids is obtained with a value of $k$ of about 0.7 . For Hildas, the dynamics is different and the resonance affects the evolution of the $\tilde{\omega}$ s. However, a forced component on the eccentricity is still present and, by looking at Fig. 3, we notice a similar clustering for the $\tilde{\omega}$ s. This behaviour has been confirmed at different epochs from the results of the numerical integration for the libration amplitude determination (see next section). The same function $\mathcal{L}$ has been used to fit the $\tilde{\omega}$ distribution and the best match is obtained with the same value of $k=0.7$ obtained for Main Belt asteroids.

After the introduction of the correcting function $\mathcal{L}$ with $k=0.7$ in the statistical algorithm we observed a reduction of about $15 \%$ in the impact probability of Hildas vs. Main Belt asteroids and an increase of approximately the same amount, $14 \%$, in the impact probability of Hildas vs. Hildas. For Hildas vs. Main Belt asteroids, the $\tilde{\omega}$ clustering shortens the number of available Main Belt projectiles for Hilda targets. As a consequence, in spite of the reduction of the available phase space for encounters, the probability of collision is lower. When we consider Hildas vs. Hildas, since projectiles and targets belong to the same population, the number of projectiles and targets remain the same, the whole Hilda population, and the clustering only reduces the available room in the phase space and increases the collision rate. 
It is worthwhile to note that the present formalism with $k>1$ might be extended also to deal the cases of librators and resonant orbits discussed in Sect. 2.1. The function $P_{A}$ there defined is very similar to $\mathcal{L}$ with suitable values of $A$ and $k$, and the results are expected to be identical.

\section{Hildas, Trojans, and SPCs}

We extracted from the Bowell's data base (Bowell et al. 1993) the orbital parameters of 232 Hilda asteroids and we computed the libration amplitudes, averaged over a period of $10^{6} \mathrm{yr}$, for each of them with a fast numerical calculation. The integration of the equation of motion for the full Solar System (all nine planets were included) plus the Hildas has been performed with the SWIFT symplectic integrator (Levison \& Duncan 1994). This sample of 232 objects is about 6 times more numerous than that adopted by Dahlgren (1998) who used 40 Hilda asteroids in his numerical model, those larger than $50 \mathrm{~km}$ in diameter. We first validated our statistical approach by computing the values of impact probability and velocity in the same sub-sample of 40 asteroids used by Dahlgren. We compare in Table 1 our values with those of Dahlgren. A good agreement is observed in all the three cases: Hildas vs. Hildas (HH), Hildas vs. Trojans (HT), and Hildas vs. Main Belt asteroids (HM). The errors reported in the Table for the impact probability computed with our statistical method are related to the numerical noise in the computation of the Montecarlo integrals (Dell'Oro \& Paolicchi 1998). These errors have not to be compared to the Dahlgren's uncertainties that are derived by the fit to the number of close encounters from the numerical data. On the other hand, the errors associated to the average impact velocity in Table 1 are fully comparable to those of Dahlgren, since they are in both cases standard deviations derived from the impact velocity distributions.

In Table 2 and Fig. 4 we give the updated values of impact probability and velocity derived from the complete sample of 232 Hilda asteroids and, at the same time we compare them to the values we computed from the small sample of 40 objects used by Dahlgren. Differences are within $5 \%$ between the corresponding values of probability and impact speed. This proves that the orbits of Hilda asteroids are well sampled in the phase space and that the group of 40 orbits was already representative of the collisional behaviour of this asteroidal population. In Fig. 4 we illustrate the distribution of the impact velocities from which the mean values of Table 2 are derived.

A part from mutual collisions and collisions with Hilda asteroids, Trojans can also encounter short period comets. These comets frequently intersect the orbit of Jupiter and they cross both the Trojan swarms. No previous estimate of the collision probability between SPC and Trojans is available, so we used our statistical model to compute it together with the impact velocity that is expected to be high due to the large eccentricity and inclination (some are retrograde) of cometary orbits. We considered two sets of cometary orbits: the C200 (JPL 2000) including comets with period lower than 200 yrs ( 185 objects), and the C20 (JPL 2000) including comets with period lower than $20 \mathrm{yrs}$ (164 objects). In Table 3 and Fig. 5 we report the collision probabilities and the impact velocity distribution. The value of $\left\langle P_{i}\right\rangle$ is more than a factor ten lower compared to the $\left\langle P_{i}\right\rangle$ of Trojans vs. Trojans and the impact velocity SPC vs. Trojan is about 30\% higher than that Trojan vs. Trojan. Taking into account that the expected number of SPCs' larger than $1 \mathrm{~km}$ is about $10^{4}$ (Fernandez et al. 1999) while the extrapolated number of Trojans is about $10^{5}$ (Marzari et al. 1997), only 1 every 100 collisions involving a Trojan asteroid is expected to be with a SPC. SPCs contribute only marginally to the collisional evolution of Trojan asteroids, although a few families in the Trojan clouds could have members of cometary origin.

\section{Kuiper Belt objects}

Collisions in the Kuiper Belt are the main source of SPCs due to the constant injection of collisional fragments into mean motion resonances. An earlier estimate of $\left\langle P_{i}\right\rangle$ and $\left\langle V_{i}\right\rangle$, based on the knowledge of TNOs available at that time (16 objects), is given in Davis \& Farinella (1997). After that paper, numerous surveys have discovered new Kuiper Belt objects increasing their known number by more than an order of magnitude. Two distinct dynamical groups have been identified: the Plutinos (46 objects) locked in the 3:2 resonance with Neptune, and standard TNOs not in resonance (140 objects). In Fig. 6 we can clearly distinguish the two groups in the $a-e$ and $a-\sin I$ plots. For the Plutinos, we computed the libration amplitudes of the critical argument $\sigma=3 \lambda-2 \lambda_{\mathrm{N}}-\tilde{\omega}$ around $180^{\circ}$ to determine the $\Delta$ function in the statistical formalism. The mean value of the semiamplitude of libration is about $83^{\circ}$ with a standard deviation of $39^{\circ}$.

A critical aspect in trying to derive reliable values of the probability of collision and average impact speed for TNOs is the presence of a strong observational bias in the data combined to a large uncertainty in the computed orbital elements due to short observational arcs. If one looks at Fig. 6, he can notice that the Plutinos are better defined in this respect since confined within the resonance. The TNOs outside the resonance cover a large interval in semimajor axis and the bias effects are unpredictable. We tried to de-bias this population with a simple algorithm: we adopted a uniform distribution of bodies with $a$ between $40 \mathrm{AU}$ and $48 \mathrm{AU}$, and $e$ between 0 and 0.15 . The inclinations have instead been extrapolated by assuming an exponential distribution $\propto \exp (B x)$. The value $B=-5.5 \pm 1$ was obtained as best fit. The inclinations have been truncated at $\sin I=0.6$. The representative sample obtained with the previous constraints was of 1000 objects that have been used to compute $\left\langle P_{i}\right\rangle$ and $\left\langle V_{i}\right\rangle$. In Table 4 we summarize the collision probabilities in the TNOs groups and in Fig. 7 we show the impact velocity distributions. 
Table 1. Comparison between the values of intrinsic probability and mean velocity of impact computed with our statistical algorithm and those computed by Dahlgren (1998). The values derived by Dahlgren are indicated with the subscript $D l$. The same sample of Hilda asteroids and of Trojan asteroids are used in the computations (bodies larger than $50 \mathrm{~km}$ in diameter)

\begin{tabular}{|c|c|c|c|c|}
\hline Group & $\left\langle P_{i}\right\rangle$ & $\left\langle P_{i}\right\rangle_{D l}$ & $\langle V\rangle$ & $\langle V\rangle_{D l}$ \\
& $\times 10^{-18} \mathrm{yr}^{-1} \mathrm{~km}^{-2}$ & $\times 10^{-18} \mathrm{yr}^{-1} \mathrm{~km}^{-2}$ & $\mathrm{~km} \mathrm{~s}^{-1}$ & $\mathrm{~km} \mathrm{~s}^{-1}$ \\
\hline $\mathrm{HH}$ & $2.02 \pm 0.01$ & $2.31 \pm 0.10$ & $3.14 \pm 1.40$ & $3.09 \pm 1.47$ \\
$\mathrm{HT}$ & $0.24 \pm 0.01$ & $0.24 \pm 0.06$ & $4.38 \pm 1.75$ & $4.59 \pm 1.71$ \\
$\mathrm{HM}$ & $0.63 \pm 0.01$ & $0.62 \pm 0.04$ & $4.61 \pm 1.70$ & $4.78 \pm 1.78$ \\
\hline
\end{tabular}

Table 2. New values for the intrinsic probability and mean velocity using 232 Hilda orbits. These values are compared with those derived from the sample of 40 asteroids

\begin{tabular}{|c|c|c|c|c|}
\hline Group & $\left\langle P_{i}\right\rangle_{232}$ & $\left\langle P_{i}\right\rangle_{40}$ & $\langle V\rangle_{232}$ & $\langle V\rangle_{40}$ \\
& $\times 10^{-18} \mathrm{yr}^{-1} \mathrm{~km}^{-2}$ & $\times 10^{-18} \mathrm{yr}^{-1} \mathrm{~km}^{-2}$ & $\mathrm{~km} \mathrm{~s}^{-1}$ & $\mathrm{~km} \mathrm{~s}^{-1}$ \\
\hline $\mathrm{HH}$ & $1.93 \pm 0.01$ & $2.02 \pm 0.01$ & $3.36 \pm 1.52$ & $3.14 \pm 1.40$ \\
$\mathrm{HT}$ & $0.27 \pm 0.01$ & $0.24 \pm 0.01$ & $4.49 \pm 1.68$ & $4.38 \pm 1.75$ \\
$\mathrm{HM}$ & $0.66 \pm 0.01$ & $0.63 \pm 0.01$ & $4.80 \pm 1.77$ & $4.61 \pm 1.70$ \\
\hline
\end{tabular}

Table 3. Probability and velocity of impact for the collisions among Trojan asteroids in the L4 and L5 swarms and the shortperiod comets.

\begin{tabular}{|c|c|c|}
\hline Group & $\left\langle P_{i}\right\rangle$ & $\langle V\rangle$ \\
& $\times 10^{-19} \mathrm{yr}^{-1} \mathrm{~km}^{-2}$ & $\mathrm{~km} \mathrm{~s}^{-1}$ \\
\hline L4-C20 & $3.70 \pm 0.01$ & $6.67 \pm 2.30$ \\
L5-C20 & $3.88 \pm 0.01$ & $6.56 \pm 2.27$ \\
\hline L4-C200 & $3.33 \pm 0.01$ & $6.78 \pm 2.71$ \\
L5-C200 & $3.48 \pm 0.01$ & $6.67 \pm 2.59$ \\
\hline
\end{tabular}

The intrinsic probability of collision obtained by Davis \& Farinella (1997) in the inner Kuiper Belt (between 42$45 \mathrm{AU}$ ) ranges from $6.210^{-22}$ to $4.2110^{-21} \mathrm{~km}^{-2} \mathrm{yr}^{-1}$, significantly higher respect to our values reported in Table 4. Also the mean impact velocities are markedly different: from 0.28 to $0.81 \mathrm{~km} \mathrm{~s}^{-1}$ those computed by Davis and Farinella, a factor two higher, from 1.08 to $1.44 \mathrm{~km} \mathrm{~s}^{-1}$, those computed by us. The discrepancy must be mainly attributed to the differences in the representative populations. In Davis \& Farinella (1997) the maximum inclination of the orbits is $\sin I=0.12$, while in our sample the higher inclination is $\sin I=0.6$. This determines a lower probability of collision and, on the other side, a higher impact velocity (higher mutual inclinations).

For the Plutinos, not included in the Davis \& Farinella (1997) analysis since at that time they were not known as a distinct population, the probability of collision has been computed taking into account the libration of the critical argument $\sigma=2 \lambda_{\mathrm{N}}-3 \lambda-\tilde{\omega}$ around $180^{\circ}$. We used the available population of 46 objects in the computations since the population seems to be well sampled in the orbital space. The values of $\left\langle P_{i}\right\rangle$ and $\langle V\rangle$ are comparable to those of the TNO population not in resonance. If the behaviour of Plutinos is similar to that of Hildas, we expect that their values of $\left\langle P_{i}\right\rangle$ and $\langle V\rangle$ will not change significantly in the future when more orbital data will be available. 

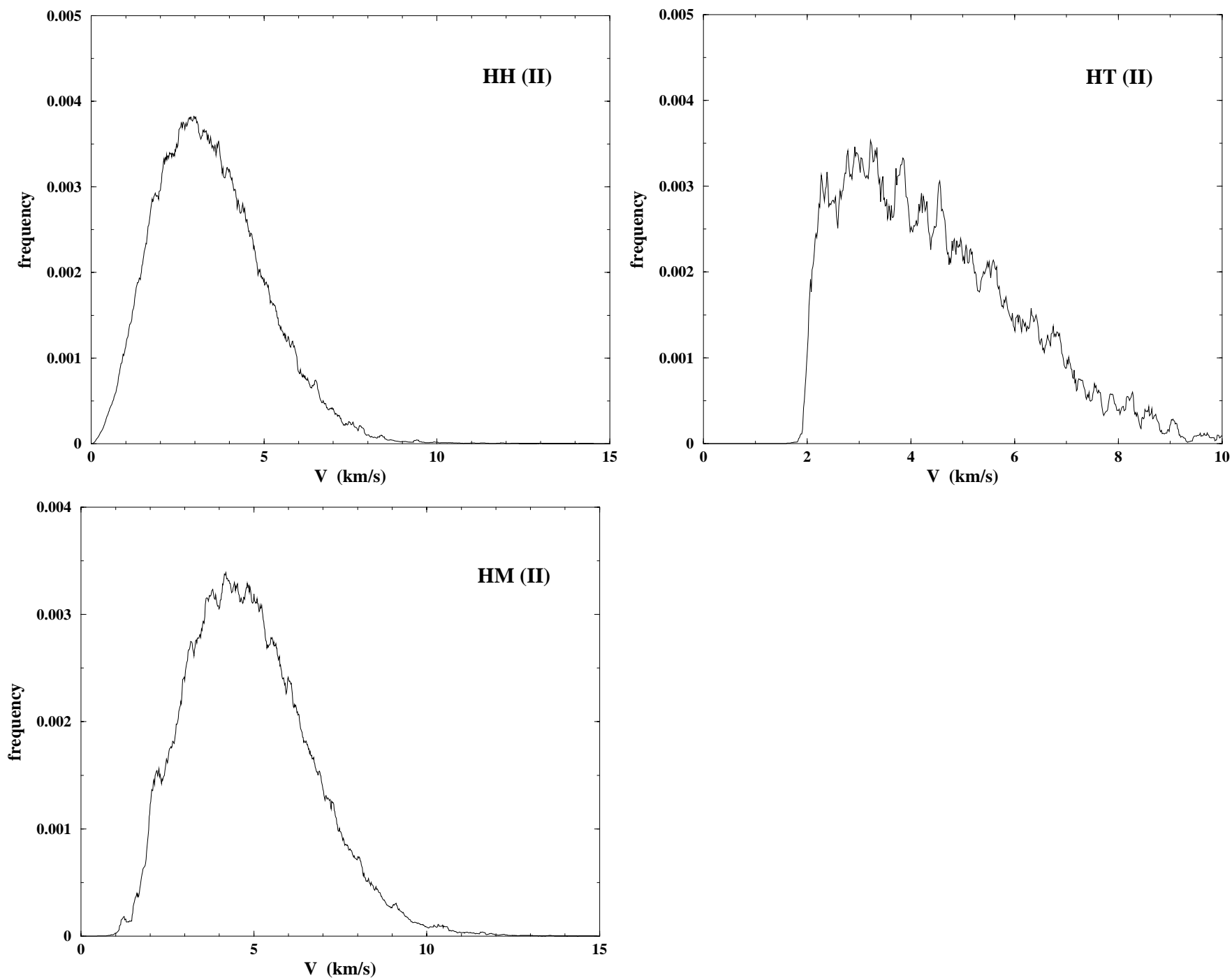

Fig. 4. Hildas: distribution of the impact velocity
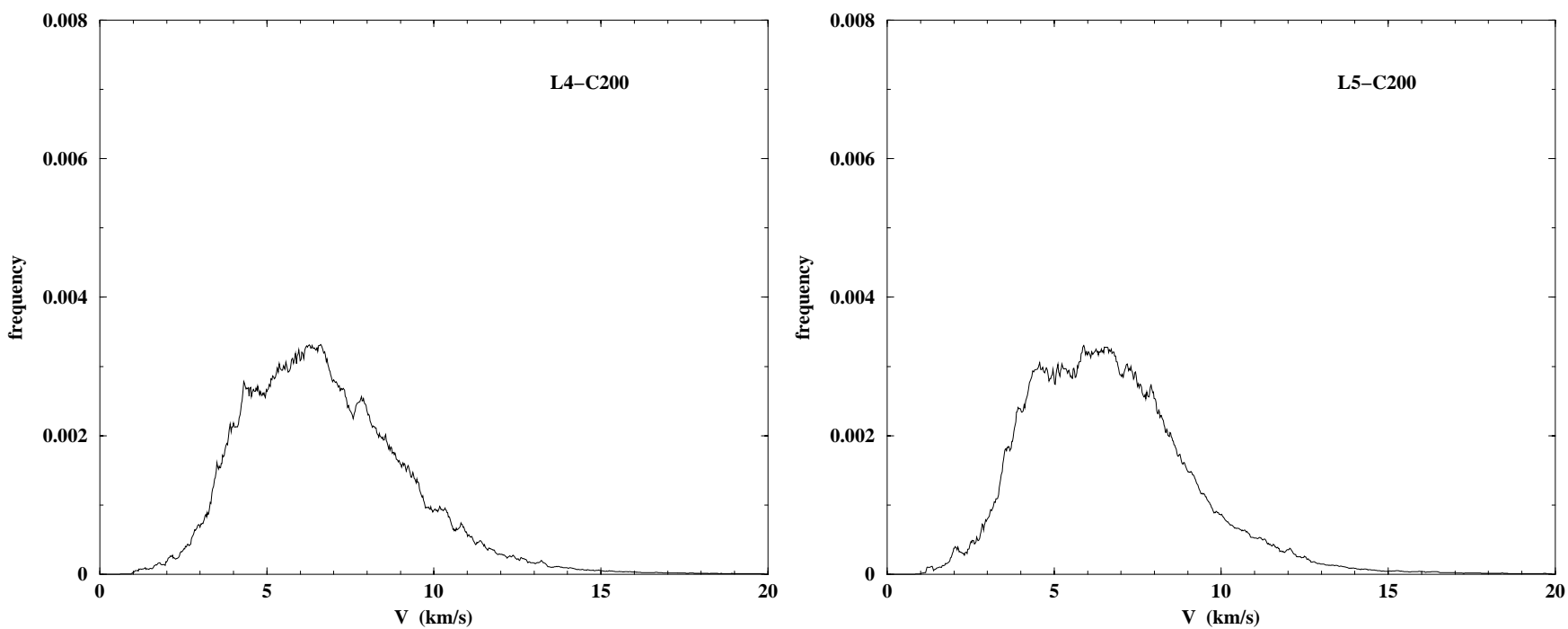

Fig. 5. Distribution of the impact velocity for the collisions among trojans asteroids and short-period comets 

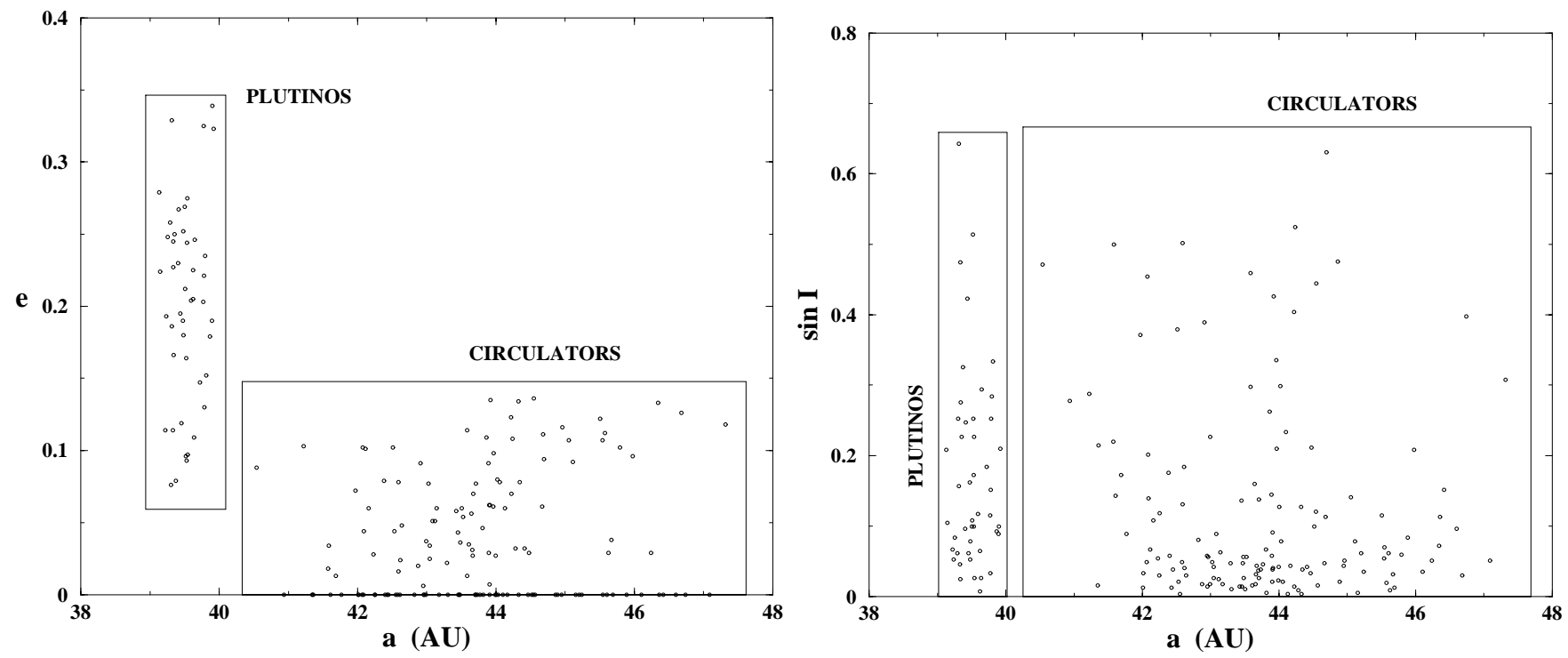

Fig. 6. TNO groups

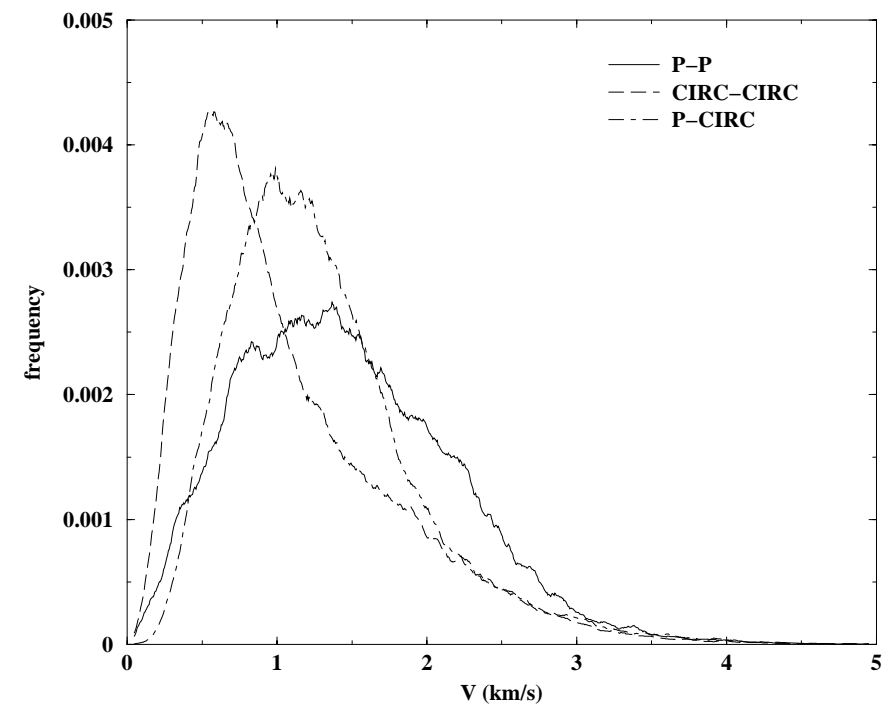

Fig. 7. Distributions of the impact velocity of the TNOs: Plutinos vs. Plutinos (P-P), circulators vs. circulators (CIRCCIRC), and plutinos vs. circulators (P-CIRC)

Acknowledgements. We are grateful to D. R. Davis for useful suggestions. The manuscript has also benefited from the comments of the referee R. Greenberg. The research has been supported by MURST-COFIN98 funding.

\section{References}

Bottke, W. F., Nolan, M. C., Greenberg, R., \& Kolvoord, R. A. 1994, Icarus, 107, 255

Bowell, E., Muinonen, K., \& Wasserman, L. H. 1993, in Asteroids, Comets, Meteors, 1994 IAU, ed. A. Milani, et al., 477

Dahlgren, M. 1998, A\&A, 336, 1056
Table 4. Probability and velocity of impact for the collisions among TNO groups

\begin{tabular}{|c|c|c|}
\hline Group & $\left\langle P_{i}\right\rangle$ & $\langle V\rangle$ \\
& $\times 10^{-22} \mathrm{yr}^{-1} \mathrm{~km}^{-2}$ & $\mathrm{~km} \mathrm{~s}^{-1}$ \\
\hline P-P & $4.44 \pm 0.04$ & $1.44 \pm 0.71$ \\
CIRC-CIRC & $3.74 \pm 0.01$ & $1.08 \pm 0.69$ \\
P-CIRC & $3.14 \pm 0.01$ & $1.23 \pm 0.62$ \\
\hline
\end{tabular}

Davis, D. R., \& Farinella, P. 1997, Icarus, 125, 50 Dell'Oro, A., Paolicchi, P., Marzari, F., Dotto, E., \& Vanzani, V. 1998, A\&A, 339, 272

Dell'Oro, A., \& Paolicchi, P. 1998, Icarus, 136, 328

Farinella, P., \& Davis, D. R. 1992, Icarus, 971, 111

Fernandez, J. A., Tancredi, G., Rickman, H., \& Licandro, J. 1999, A\&A, 352, 327

Greenberg, R. 1975, MNRAS, 170, 295

Greenberg, R. 1977, Vistas Astron., 21, 209

Greenberg, R. 1982, AJ, 87, 185

JPL 2000, JPL's solar system dynamics database, WWW site: ssd.jpl.nasa.gov

Kresák, L. 1967, Bulletin of the Astronomical Institute of Czechoslovakia, 18, 27

Levison, H., \& Duncan, M. 1994, Icarus, 108, 18

Marzari, F., Scholl, H., \& Farinella, P. 1996, Icarus, 119, 192

Marzari, F., Farinella, P., Davis, D. R., Scholl, H., \& Campo Bagatin, A. 1997, Icarus, 125, 39

Vedder, J. D. 1996, Icarus, 123, 436

Wetherill, G. W. 1967, J. Geophys. Res., 72, 2429 